\title{
Eosinophils and Trichinella infection: toxic for the parasite and the host?
}

\author{
Fabrizio Bruschi ${ }^{1}$, Masataka Korenaga ${ }^{2}$ and Naohiro Watanabe ${ }^{3}$ \\ ${ }^{1}$ Department of Experimental Pathology, Medical Biotechnologies, Infectivology and Epidemiology, Università di Pisa, \\ Medical School, Via Roma 55, 56126 Pisa, Italy \\ ${ }^{2}$ Department of Parasitology, Kochi Medical School, Kochi University, Nankokushi, Kochi 783-8505, Japan \\ ${ }^{3}$ Department of Tropical Medicine, Jikei University School of Medicine, 3-25-8 Nishi-shinbashi, Minatoku, Tokyo \\ 105-8461, Japan \\ Corresponding author. Bruschi, F. (fbruschi@med.unipi.it).
}

Peripheral blood and tissue eosinophilia characterize trichinellosis in humans, and present in addition to the increased total IgE levels that occur in many helminth infections. Both processes are the consequence of T-helper 2 activation. Blood and tissue eosinophilia begins with eosinophilopoiesis in the bone marrow, which is followed by the migration of eosinophils through the circulatory system, the eosinophil infiltration of tissues at the inflammatory foci and, finally, degranulation and cell death. Recently, some aspects of eosinophilia caused by Trichinella spiralis infection have been elucidated; however, the protective role of this population of cells against Trichinella parasites remains controversial. Furthermore, when eosinophils are numerous, they can be toxic for host tissues. This review discusses these issues in both human and rodent infection models.

\section{Eosinophilopoiesis}

Trichinellosis is a worldwide zoonosis, and there are an estimated ten million people at risk for this parasitic infection [1]. The life cycle of Trichinella parasites consists of the enteral phase, which lasts 3-4 weeks in humans, and the parenteral phase, which starts after the arrival of migrant larvae in the striated skeletal muscle fibers. At the beginning of the parenteral phase, an increase in blood and tissue eosinophil levels is observed [2].

Trichinella antigens stimulate $\mathrm{T}$ cells to produce cytokines that induce eosinophilopoiesis in the infected host [3,4] (Figure 1). When spleen lymphocytes that were obtained from mice 4-6 weeks after infection with Trichinella spiralis were cultured with somatic antigens [5] or excretory and/or secretory products from muscle larvae (ML) [6], the conditioned medium induced eosinophil production from normal, nonadherent syngeneic bone-marrow cells. Eosinophilic responses in Trichinella-infected mice were dependent on the release of soluble products from lymphocytes that were sensitized to the parasite antigen, whereas the addition of the antigen to either non-sensitized lymphocytes or lymphocytes sensitized by the Calmette-Guérin bacillus did not induce colony-stimulating activity [5]. However, a direct injection of the antigen failed to induce eosinophilia in rats [7].

Interleukin-5 (IL-5) is an important cytokine that is involved in eosinophilopoiesis in mice that are infected with T. spiralis [8] (Table 1). Immunization with $\mathrm{ML}$ antigens combined with adjuvant induces a type-2-biased cytokine response and generates a high level of IL-5 [9-11]. Wang et al. [12] suggested that the interactions between antigen-presenting cells and $\mathrm{CD}^{+} \mathrm{T}$ cells through co-stimulatory CD86 are crucial to IL-5 production in $T$. spiralis infections. The intraperitoneal injection of Trichinella-infected BALB/c mice with anti-CD80 and/or anti-CD86 monoclonal antibody (moAb) suppressed eosinophilia. The combination of both moAbs also effectively reduced the in vitro production of IL-5 in mesenteric lymph node cells that were stimulated with somatic antigen; however, when further studied, the in vitro production of IL- 5 was only reduced in the presence of anti-CD86 where anti-CD80 alone was ineffective. When co-stimulatory factor interactions between intercellular adhesion molecule- 1 and lymphocyte function antigen-1, or vascular cell adhesion molecule (VCAM) and very late antigen-4 were blocked in vitro, the production of IL-5 was not suppressed as effectively as it was in anti-CD86 moAb treatment.

\section{Eosinophil migration and infiltration to tissues}

Although homogenates of $T$. spiralis ML attract eosinophils [13], the migration of eosinophils into tissues is also the result of the action of chemokines, in particular the CCR3 ligands; adhesion molecules, especially those mediated by P-selectin and $\alpha 4$ integrins; and IL-5. CCR3 expressed on eosinophils in humans and mice mediates both chemotaxis and adhesion to endothelial cells in vitro and in vivo. Although CCR3 deficiency does not affect the capacity of mice to generate peripheral blood eosinophilia in response to $T$. spiralis, eosinophils are not recruited to the jejunal mucosa, nor are they present in the striated muscle adjacent to encysted larvae [14]. P-selectin mediates IL-13-induced eosinophil transmigration but not eotaxin production in mice [15]; however, the regulatory role of P-selectin in eosinophil transmigration during T. spiralis infection is unclear.

The trafficking of leukocytes to the gut-associated lymphoid tissue depends on the interaction of the $\alpha 4 \beta 7$ integrin with its ligand, the mucosal vascular addressin cell-adhesion molecule-1 (MAdCAM-1) that is present on 
the endothelium [16]. Both eosinophils and mast cells express $\alpha 4 \beta 7 \quad[17,18]$. During T. spiralis infection, intestinal eosinophilia was delayed and reduced in $\beta 7$-integrin-deficient mice but not in mice that were treated with moAb to integrin $\beta 7$, whereas the suppression of mastocytosis was observed in both experimental models $[19,20]$. These facts indicate that the contribution of $\beta 7$ integrin to eosinophil tissue migration is inferior to that of the mast cells. Galectin-3 (Gal-3) has a crucial role in eosinophil recruitment and airway allergic inflammation of mice [21]. In humans, eosinophils from allergic donors express elevated levels of Gal-3, which co-localizes with $\alpha$ integrin and increase the rolling and firm adhesion of eosinophils on the VCAM-1 of endothelial cells [22].

Eotaxin is a fundamental regulator of physiological eosinophil trafficking during healthy states [23] and inflammation [24]. Ecalectin (galectin-9), which is produced by antigen-stimulated $\mathrm{T}$ cells, is a powerful eosinophil-specific chemoattractant in vitro and in vivo [25]. The role of these chemokines in eosinophilia that is caused by $T$. spiralis infection has been partially clarified. Eotaxin-1 (CCL11) is important in intestinal-tissue eosinophilia but not in peripheral eosinophilia during infection with $T$. spiralis [13]. In addition, eotaxin-2 (CCL24) is only induced in the infected intestine. When $\mathrm{CD} 4^{+} \mathrm{OX} 22^{-} \mathrm{T}$ cells from rats infected with $T$. spiralis were transferred to uninfected rats and followed by a challenge infection, the cells that migrated into the intestinal epithelium induced intestinal eosinophilia [26]. There is a possibility that $\mathrm{CD}^{+} \mathrm{OX} 22^{-} \mathrm{T}$ cells produce more chemokines that have chemotactic activity against eosinophils than $\mathrm{CD} 4^{+} \mathrm{OX} 22^{+} \mathrm{T}$ cells.

In other helminthic diseases, such as that induced by Brugia malayi, alternatively activated macrophages (AAM) under the control of T-helper 2 (Th2)-dependent cytokines express unique products such as Arg 1 (arginase 1), FIZZ 1 (resistin-like $\alpha$ ) and YM1 (chitinase-like molecule), the functions of which have not been fully elucidated. Furthermore, AAM and/or YM1 are involved in eosinophil chemotaxis and might stimulate the migration of cells to parasites [27,28]. Induced AAM and YM1 have been shown in both mice and guinea pigs infected with $T$. spiralis $[29,30]$.

\section{Degranulation of eosinophils}

Eosinophils contain several types of granules and secretory organelles that are packed with major basic protein, eosinophil cationic protein, eosinophil-derived neurotoxin and eosinophil peroxidase. These proteins perform various biological activities [31]. It has been suggested that the immunoglobulins IgG and IgA (including secretory IgA) are able to trigger eosinophil degranulation [31]. However, the presence of $\mathrm{IgE}$ receptors on eosinophils remains controversial [31-33]. Recently, $\mathrm{T}$ cells have also been shown to have a role in eosinophil degranulation. Rag- $1^{-1-}$ mice that lacked $\mathrm{T}$ and $\mathrm{B}$ cells were infected with Nippostrongylus brasiliensis, and IL-4-expressing eosinophils were recruited to pulmonary tissues but failed to degranulate [34]. Reconstitution with $\mathrm{CD}^{+} \mathrm{T}$ cells promoted the accumulation of degranulated IL-4-expressing eosinophils, but only if the $\mathrm{T}$ cells were stimulated with a cognate antigen. These facts indicate that T-helper cells confer antigen specificity on eosinophil cytotoxicity but not on the cytokine responses. Whether a similar mechanism is invoked during the infection with $T$. spiralis has not been studied.

Cytotoxic granules injure both the host tissue and the parasite. Eosinophils are thought to be involved in myocarditis in rats that are infected with T. spiralis [35]. Eosinophils are probably recruited by mediators, such as platelet-activating factor and eosinophil-chemotactic factor for anaphylaxis, that are released by degranulated mast cells that are activated by cognate antigen binding to $\mathrm{IgE}$.

\section{Apoptosis of eosinophils}

Gon et al. [36] demonstrated that a considerable percentage of eosinophils in non-infected rats undergo apoptosis within a few hours, whereas the apoptosis rate of eosinophils from Trichinella-infected rats is notably lower. The inhibition of eosinophil apoptosis during the later stages of a Trichinella infection is related to the presence of IL-5. By contrast, the inhibition of eosinophil apoptosis during the earlier phases of the infection is thought to be unrelated to IL-5, possibly because of the presence of IL-3 and the granulocyte-macrophage colony-stimulating factor (GM-CSF). At 2-5 weeks post-infection, when the recovery phase of a $T$. spiralis infection occurs, excess eosinophils disappear slowly from the jejunum of mice, whereas apoptotic eosinophils remain. The draining mesenteric lymph nodes also contain large numbers of apoptotic eosinophils [37]. Therefore, it is believed that jejunal eosinophils undergo apoptosis locally once the parasite-specific $\mathrm{T}$ cells cease to be prominent in the intestine and after the adult worms have been expelled.

\section{Protection by eosinophils in murine models}

It is evident that host defenses to $T$. spiralis are mediated through the action of $\mathrm{CD}^{+}$T-cell-derived cytokines [38-42]. Studies using IL-4 or IL-4-receptor- $\alpha$ deficient mice revealed that IL- 4 has a crucial role in worm expulsion [43,44]. However, it is not clear whether IL-5 and/or eosinophils can be implicated in the protective response of the host against the parasite. Neither IL-5 nor eosinophils were essential to control the parasite in either primary or challenge infections of CF1 mice using anti-IL-5 moAb [8]. Furthermore, when non-immunized $\mathrm{C} 3 \mathrm{H} / \mathrm{HeN}$ and IL-5 transgenic ( $\mathrm{Tg}$ ) mice were compared to $\mathrm{C} 3 \mathrm{H} / \mathrm{HeN}$ and IL-5 Tg mice that were immunized with parasite somatic antigen, no differences were found in the recovery of ML or adult worms in the small intestine, the fecundity of female adult worms, or the infectivity of newborn larvae among the groups [45].

However, protective immune responses against the parasite should be analyzed carefully. Experimental models using CCR3-deficient BALB/c [14], CCL11- and IL-5-deficient BALB/c [13], and IL-5-deficient C57BL/6 mice [46] show that eosinophils are not essential for the expulsion of adult worms after a primary infection. By contrast, IL-5 and/or eosinophils are involved in the expulsion of adult worms after a secondary infection in IL-5-deficient C57BL/6 mice [47]. Worm burdens in the skeletal muscle were increased and the frequency of 
encysted larvae that exhibited necrosis was reduced in CCR3-deficient mice, compared with wild-type mice, during a primary infection [14]. This observation supports the hypothesis that eosinophils are cytotoxic versus the larval stage in mice $[48,49]$. Mouse eosinophils adhere to and kill $T$. spiralis newborn larvae in an antibody-dependent cellular cytotoxicity system [49] (Figure 2 and Figure 3).

\section{Untangling the role of eosinophils in experimental trichinellosis}

The discrepancy of the role of eosinophils in protection might be due to the mouse strains, the stages of the parasites and/or the experimental models used. Recently, strain-dependent resistance in the asthma model in mice has been reported to correlate with the apoptosis rate of lung-derived eosinophils [50]. Another possibility might be the difference in the degree of eosinophil activation. Hypodense eosinophils are commonly found in infected tissue, and cytotoxic activity against parasites is increased in this population of eosinophils [51,52]. Eosinophils can release large amounts of powerful mediators to kill parasites, injure tissues and remodel the damaged tissue. Thus, it is possible that eosinophils might be important in host defense. To validate this hypothesis, however, the interactions between each stage of the parasite and the eosinophils will require further study. In addition, a comparison of different Trichinella spp. might shed some light on host defense mechanisms [53-55]. For example, both adult worms and newborn larvae of Trichinella pseudospiralis exhibit stronger neutrophil chemotactic activity than those of $T$. spiralis [56].

\section{Eosinophilia in humans}

Eosinophilia is present, with few exceptions, in most cases of human trichinellosis, inasmuch as it is the earliest and most important host response. Even in asymptomatic cases, increases in eosinophilia of up to $15 \%$ have been observed [2].

The mechanisms of eosinophil production in humans are similar to those described in experimental animals. Recently, a type-2 cytokine pattern of lymphocyte activation has been described during the muscular phase of infection in patients that are infected by either T. spiralis or Trichinella britovi [57]. Type- 2 cytokines were elevated during the first nine months of infection. In particular, IL-5 mRNA expression and protein production were both overproduced in peripheral blood mononuclear cells that were stimulated with $T$. spiralis or $T$. britovi antigens. The increased mRNA expression lasted at least one year; however, IL-5 protein levels peaked at two months and declined sharply after, even though considerable levels remained after 14 months [58].

Eosinophilia appears at an early stage of infection, before the development of general clinical signs and symptoms, and it increases between the second and fifth weeks of infection [59]. Eosinophilia might be low $(<1000$ cells per $\mu \mathrm{l})$, moderate $(1000-3000$ cells per $\mu \mathrm{l})$ or high (>3000 cells per $\mu \mathrm{l}$, and up to 19000 cells per $\mu \mathrm{l}$ have been reported) [2]. Eosinophilia declines slowly and remains at low levels for several weeks, up to three months post-infection. The level of eosinophilia generally correlates with the degree of myalgia [60] and is significantly higher in people with neurological complications (i.e. neurotrichinosis). In fact, eosinophilia presenting with more than 4000 cells per $\mu \mathrm{l}$ was observed in $87.5 \%$ of patients with cerebral damage and in only $35.2 \%$ of those with no central neurological complications [61].

A significant correlation was observed between the presence of specific $\operatorname{IgE}$ in the patient sera during the first period of infection and blood eosinophilia levels. In fact, $73 \%$ of patients with blood eosinophilia after four months of infection were positive for specific IgE compared to only $28 \%$ of patients without eosinophilia [61]. During the acute stage of infection, a massive decrease of eosinophils in people with severe trichinellosis is a reasonable warning of a severe outcome, and a sudden decrease to $1 \%$ or less might even predict patient death [2]. The mechanism underlying this phenomenon is not known but is likely to be related to a massive peripheral migration.

Human eosinophils could have a protective role because they are cytotoxic against newborn larvae in an antibody-dependent cellular cytotoxicity system [62]. Whether eosinophils are more cytotoxic than neutrophils is controversial. It is difficult to say whether the cytotoxic ability of these cells can have a meaningful role in protection against the parasite. The observation that eosinophil number decreases massively in severe infections [59] is in favor of a protective role in vivo as well.

There is no doubt that the increase in activated eosinophil levels is responsible for damage to the vascular walls (probably because of the release of the major basic protein, which is elevated in patients with eosinophilia [63]), and this explains tissue damage in the central nervous system (CNS) [60] and other tissues. Figure 4 shows a rich eosinophil infiltrate in the myocardial tissue of one patient who died because of severe trichinellosis. There is a correlation between eosinophil levels and muscle enzyme (creatine phosphokinase and lactate dehydrogenase) levels in skeletal muscles, and between eosinophil levels and myalgic score in patients infected by $T$. britovi; this indicates a relationship between eosinophil levels, and tissue damage and pain [59].

\section{Concluding remarks}

Clinical observations indicate that allergic manifestations are typical of human trichinellosis. At the same time, eosinophils typically increase in number during infection. Is this phenomenon a side-effect of Th2 activation, or can these cells kill parasites in vivo and in vitro? The rapid decrease in eosinophil levels as a predictor of patient death is consistent with a protective role for eosinophils in humans.

However, a chronic exposure to activated eosinophils might cause tissue damage in the muscle, myocardium and CNS, as exemplified in the most severe form of human trichinellosis, the so-called 'neurotrichinosis'. Further studies are needed at a basic level to improve knowledge about mechanisms of eosinophil neurotoxicity.

Despite the lack of controlled studies, corticosteroids are used by infectious-disease specialists to prevent immediate-type reactions during trichinellosis. 
Corticosteroids should always be used in combination with anthelmintics to prevent an increased worm burden caused by delayed worm expulsion. Such anti-inflammatory drugs are also used to treat vasculitis, owing to its effects in inhibiting eosinophil activation and degranulation [31].

\section{Acknowledgements}

We would like to thank Dante Zarlenga for his critical reading of the manuscript and Y. Hashiguchi, Kochi Medical School for his encouragement in this work. A part of this study was supported by the grant-in-aid for scientific research from the Japanese Ministry of Education, Science, Technology, Culture and Sport (no. 14570216) and by the Uehara Memorial Foundation. F.B. dedicates this article to Gino Malvaldi, to acknowledge the support and advice received during his career.

\section{References}

1 Dupouy-Camet, J. (2000) Trichinellosis: a worldwide zoonosis. Vet. Parasitol. 93, 191-200

2 Dupouy-Camet, J. et al. (2002) Opinion on the diagnosis and treatment of human trichinellosis. Expert Opin. Pharmacother. 3, $1117-1130$

3 Basten, A. and Beeson, P.B. (1970) Mechanism of eosinophilia II. Role of the lymphocyte. J. Exp. Med. 131, 1288-1305

4 Walls, R.S. et al. (1973) The immunopathology of trichiniasis in T-cell deficient mice. Clin. Exp. Immunol. 13, 231-242

5 Ruscetti, F.W. et al. (1976) Specific release of neutrophilicand eosinophilic-stimulating factors from sensitized lymphocytes. Blood 47, 757-765

6 Bartelmez, S.H. et al. (1980) Differential regulation of spleen cell-mediated eosinophil and neutrophil-macrophage production. Blood 55, 489-493

7 Basten, A. et al. (1970) Mechanism of eosinophilia. I. Factors affecting the eosinophil response of rats to Trichinella spiralis. J. Exp. Med. 131, 1271-1287

8 Herndon, F.J. and Kayes, S.G. (1992) Depletion of eosinophils by anti-IL-5 monoclonal antibody treatment of mice infected with Trichinella spiralis does not alter parasite burden or immunologic resistance to reinfection. J. Immunol. 149, 3642-3647

9 Robinson, K. et al. (1994) Vaccination against the nematode Trichinella spiralis in high- and low-responder mice. Effects of different adjuvants upon protective immunity and immune responsiveness. Immunology 82, 261-267

10 Robinson, K. et al. (1995) Immune response profiles in vaccinated and non-vaccinated high- and low-responder mice during infection with the intestinal nematode Trichinella spiralis. Parasitology 110, 71-78

11 McGuire, C. et al. (2002) Nasal immunization with homogenate and peptide antigens induces protective immunity against Trichinella spiralis. Infect. Immun. 70, 7149-7152

12 Wang, Z. et al. (2000) Costimulatory signal through CD86 is important in Th2 response in Trichinella spiralis infected mice. Parasite Immunol. 22, 121-130

13 Dixon, H. et al. (2006) The role of Th2 cytokines, chemokines and parasite products in eosinophil recruitment to the gastrointestinal mucosa during helminth infection. Eur. J. Immunol. 36, 1753-1763

14 Gurish, M.F. et al. (2002) CCR3 is required for tissue eosinophilia and larval cytotoxicity after infection with Trichinella spiralis. J. Immunol. 168, 5730-5736

15 Larbi, K.Y. et al. (2003) P-selectin mediates IL-13-induced eosinophil transmigration but not eotaxin generation in vivo: a comparative study with IL-4-elicited responses. J. Leukoc. Biol. 73, $65-73$

16 Berlin, C. et al. (1993) $\alpha 4 \beta 7$ integrin mediates lymphocytes binding to the mucosal vascular addressin MAdCAM-1. Cell 74, 185-195

17 Gurish, M.F. et al. (1992) Expression of murine $\beta 7, \alpha 4$ and $\beta 1$ integrin genes by rodent mast cells. J. Immunol. 149, 1964-1972 18 Walsh, G.M. et al. (1996) Integrin $\alpha 4 \beta 7$ mediates human eosinophil interaction with MAdCAM-1, VCAM-1 and fibronectin. Immunology 89, 112-119

19 Artis, D. et al. (2000) $\beta 7$ integrin-deficient mice: delayed leukocyte recruitment and attenuated protective immunity in the small intestine during enteric helminth infection. Eur. J. Immunol. 30, 1656-1664

20 McDermott, J.R. et al. (2001) Leukocyte recruitment during enteric nematode infection. Immunology 103, 505-510

21 Zuberi, R.I. et al. (2004) Critical role for Galectin-3 in airway inflammation and bronchial hyperresponsiveness in a murine model of asthma. Am. J. Pathol. 165, 2045-2053

22 Rao, S.P. et al. (2007) Galectin-3 functions as an adhesion molecule to support eosinophil rolling and adhesion under conditions of flow. J. Immunol. 179, 7800-7807

23 Matthews, A.N. et al. (1998) Eotaxin is required for the baseline level of tissue eosinophils. Proc. Natl. Acad. Sci. U. S. A. 95, 6273-6278

24 Ganzalo, J.A. et al. (1996) Mouse eotaxin expression parallels eosinophil accumulation during lung allergic inflammation but it is not restricted to a Th2-type response. Immunity 4, 1-14

25 Hirashima, M. (1999) Ecalectin as a T cell-derived eosinophil chemoattractant. Int. Arch. Allergy Immunol. 120 (Suppl. 1), 7-10 26 Wang, C.H. et al. (1990) T helper subset function in the gut of rats: differential stimulation of eosinophils, mucosal mast cells and antibody forming cells by OX8-OX22+ and OX8-OX22-Immunology $71,166-175$

27 Voehringer, D. et al. (2007) Eosinophils develop in distinct stages and are recruited to peripheral sites by alternatively activated macrophages. J. Leukoc. Biol. 81, 1434-1444

28 Maizels, R.M. et al. (2004) Helminth parasites - masters of regulation. Immunol. Rev. 201, 89-116

29 Chang, N-C.A. et al. (2001) A macrophage protein, Ym1, transiently expressed during inflammation is a novel mammalian lectin. J. Biol. Chem. 276, 17497-17506

30 Dzik, J.M. et al. (2004) A non-classical type of alveolar macrophage response to Trichinella spiralis infection. Parasite Immunol. 26, 197-205

31 Hogan, S.P. et al. (2008) Eosinophils: biological properties and role in health and disease. Clin. Exp. Allergy 38, 709-750

32 Gounni, A.S. et al. (1994) High-affinity $\operatorname{IgE}$ receptor on eosinophils is involved in defense against parasites. Nature 367, 183-186

33 Gould, H.J. et al. (2003) The biology of IgE and basis of allergic disease. Annu. Rev. Immunol. 21, 579-628

34 Shinkai, K. et al. (2002) Helper T cells regulate type-2 innate immunity in vivo. Nature 420, 825-829

35 Paolocci, N. et al. (1998) Immunopathological mechanisms underlying the time-course of Trichinella spiralis cadiomyopathy in rats. Virchows Arch. 432, 261-266

36 Gon, S. et al. (1997) Apoptosis and in vivo distribution and clearance of eosinophils in normal and Trichinella spiralis-infected rats. J. Leukoc. Biol. 62, 309-317

37 Friend, D.S. et al. (2000) Senescent jejunal mast cells and eosinophils in the mouse preferentially translocate to the spleen and draining lymph node, respectively, during the recovery phase of helminth infection. J. Immunol. 165, 344-352

38 Wakelin, D. and Wilson, M.M. (1979) T and B cells in the transfer of immunity against Trichinella spiralis in mice. Immunology 37, 103-109

39 Grencis, R.K. et al. (1985) L3T4-positive T lymphoblasts are responsible for transfer of immunity to Trichinella spiralis in mice. Immunology 56, 213-218

40 Bell, R.G. et al. (1987) Characterization of a cell population in thoracic duct lymph that adoptively transfers rejection of adult Trichinella spiralis to normal rats. Immunology 61, 221-227

41 Korenaga, M. et al. (1989) Intestinal immunity to Trichinella spiralis is a property of OX8-OX22- Thelper cells that are generated in the intestine. Immunology 66, 588-594

42 Ramaswamy, K. et al. (1994) cytokine profile of protective anti-Trichinella spiralis $\mathrm{CD} 4^{+} \mathrm{OX} 22$ and non-protective $\mathrm{CD} 4^{+} \mathrm{OX} 22^{+}$thoracic duct cells in rats: secretion of IL-4 alone does not determine protective capacity. Parasite Immunol. 16, 435-445 43 Lawrence, C.E. et al. (1998) IL-4-related enteropathy in an intestinal nematode infection. Eur. J. Immunol. 28, 2672-2684 44 Urban, J.F., Jr et al. (2000) Stat6 signaling promotes protective immunity against Trichinella spiralis through a mast cell- and T cell-dependent mechanism. J. Immunol. 164, 2046-2052 
45 Hokibara, S. et al. (1997) Marked eosinophilia in interleukin-5 transgenic mice fails to prevent Trichinella spiralis infection. J. Parasitol. 83, 1186-1189

46 Vallance, B.A. et al. (2000) Interleukin-5 deficient mice exhibit impaired host defence against challenge Trichinella spiralis infections. Parasite Immunol. 22, 487-492

47 Vallance, B.A. et al. (1999) IL-5 contributes to worm expulsion and muscle hypercontractility in a primary $T$. spiralis infection. Am. J. Physiol. 277, G400-G408

48 Grove, D.I. et al. (1977) Eosinophils and resistance to Trichinella spiralis. J. Exp. Med. 145, 755-759

49 Gansmüller, A. et al. (1987) Antibody-dependent in vitro cytotoxicity of newborn Trichinella spiralis larvae. Nature of the cells involved. Parasite Immunol. 9, 281-292

50 Tumes, D.J. et al. (2007) Strain-dependent resistance to allergen-induced lung pathophysiology in mice correlates with rate apoptosis of lung-derived eosinophils. J. Leukoc. Biol. 81, $1362-1373$

51 Capron, M. et al. (1984) Role of IgE receptors in effector function of human eosinophils. J. Immunol. 132, 462-468

52 Hamada, A. et al. (1992) Occurrence and characteristics of hypodense eosinophils in rats infected with Trichinella spiralis. Parasite Immunol. 14, 503-512

53 Wakelin, D. et al. (1994) Immune responses to Trichinella spiralis and T. pseudospiralis in mice. Immunology 81, 475-479

54 Furze, R.C. and Selkirk, M.E. (2005) Comparative dynamics and phenotype of the murine immune response to Trichinella spiralis and Trichinella pseudospiralis. Parasite Immunol. 27, 181-188

55 Lee, K.M. and Ko, R.C. (2006) Cell-mediated response at the muscle phase of Trichinella pseudospiralis and Trichinella spiralis infections. Parasitol. Res. 99, 70-77
56 Shupe, K. and Stewart, G.L. (1991) Stimulated chemotactic response in neutrophils from Trichinella pseudospiralis-infected mice and the neutrophilotactic potential of Trichinella extracts. Int. J. Parasitol. 21, 625-630

57 Morales, M.A. et al. (2002) Increased CD8+-T cell expression and a Type 2 cytokine pattern during the musculature phase of Trichinella infection in humans. Infect. Immun. 70, 233-239

58 Capo, V. and Despommier, D.D. (1996) Clinical aspects of infection with Trichinella spp. Clin. Microbiol. Rev. 9, 47-54

59 Ferraccioli, G.F. et al. (1988) Prospective rheumatological study of muscle and joint symptons during Trichinella nelsoni infection. Q. J. Med. 69, 973-984

60 Fourestié, V. et al. (1993) Neurotrichinosis. A cerebrovascular disease associated with myocardial injury and hypereosinophilia. Brain 116, 603-616

61 Dupouy-Camet, J. et al. (1988) Etude des immunoglobulines specifiques (totales, IgG, IgM, IgA, IgE) en immunofluorescence indirecte et en ELISA chez quarante malades trichinds suivis pendant neuf mois. Pathol. Biol. (Paris) 36, 803-807

62 Kazura, J.W. (1981) Host defense mechanisms against nematode parasites: destruction of newborn Trichinella spiralis larvae by human antibodies and granulocytes. J. Infect. Dis. 143 , $712-718$

63 Wassom, D.L. et al. (1981) Elevated serum levels of the eosinophil granule major basic protein in patients with eosinophilia. J. Clin. Invest. 67, 651-661

64 Hamann, K.J. et al. (1987) Comparative toxicity of purified human eosinophil granule proteins for newborn larvae of Trichinella spiralis. J. Parasitol. 73, 523-529 


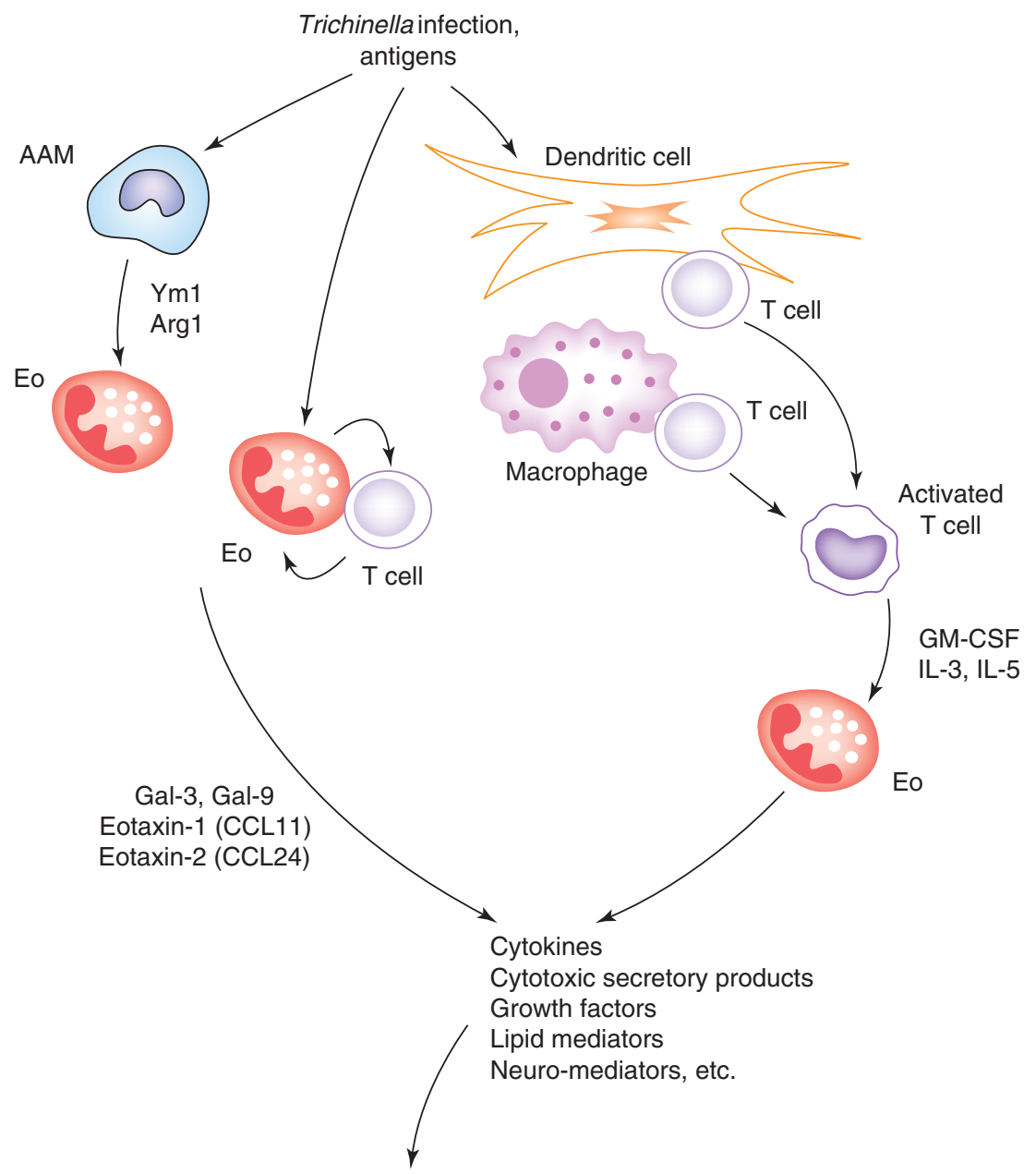

Larval killing

Tissue injury

Tissue repairing

Worm expulsion?

Figure 1. Eosinophil induction, recruitment and products during Trichinella spiralis infection. Trichinella antigens stimulate dendritic cells and classic macrophages to interact with T cells. Activated T cells produce cytokines including GM-CSF, IL-3 and IL-5. In the bone marrow, precursors proliferate and differentiate into eosinophils. Eosinophil migration from the bone marrow to the vessels is controlled mainly by IL-5. Trichinel/a antigens might attract and affect eosinophils directly to interact with T cells. AAMs also attract eosinophils through Ym1 and/or Arg1. Strong chemoattractants for eosinophils are Gal-9, eotaxin-1 and eotaxin-2. Gal-3 functions as a cell-surface adhesion molecule to support eosinophil rolling and adhesion. Eosinophils around T. spiralis in the tissues produce cytokines, cytotoxic secretory products, growth factors, lipid mediators and neuro-mediators. These molecules might be involved in larval killing, tissue injury and/or tissue repair, together with various cells. The involvement of eosinophils in worm expulsion from the gut is still obscure. Abbreviations: AAM, alternatively activated macrophage; Arg1, arginase 1; Eo, eosinophil; Gal-3, Galectin 3; Gal-9, Galectin 9; GM-CSF, granulocyte-macrophage colony-stimulating factor; IL-3, interleukin 3; IL-5, interleukin 5; YM1, chitinase-like molecule.

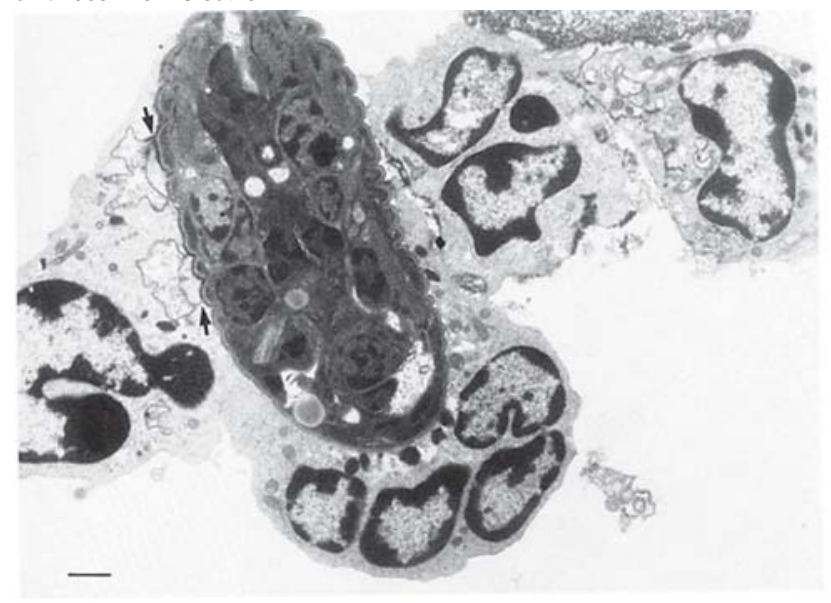

$\overline{\text { TRENDS in Parasitology }}$ 
Figure 2. Electron micrograph of eosinophils adhering to Trichinella spiralis. Eosinophils from normal mice adhering to a Trichinella spiralis newborn larva incubated with immune sera. The arrows indicate intracellular vacuoles in contact with the cell basal membrane. Scale bar represents $1.3 \mu \mathrm{m}$. Reprinted, with permission, from Ref. [49].

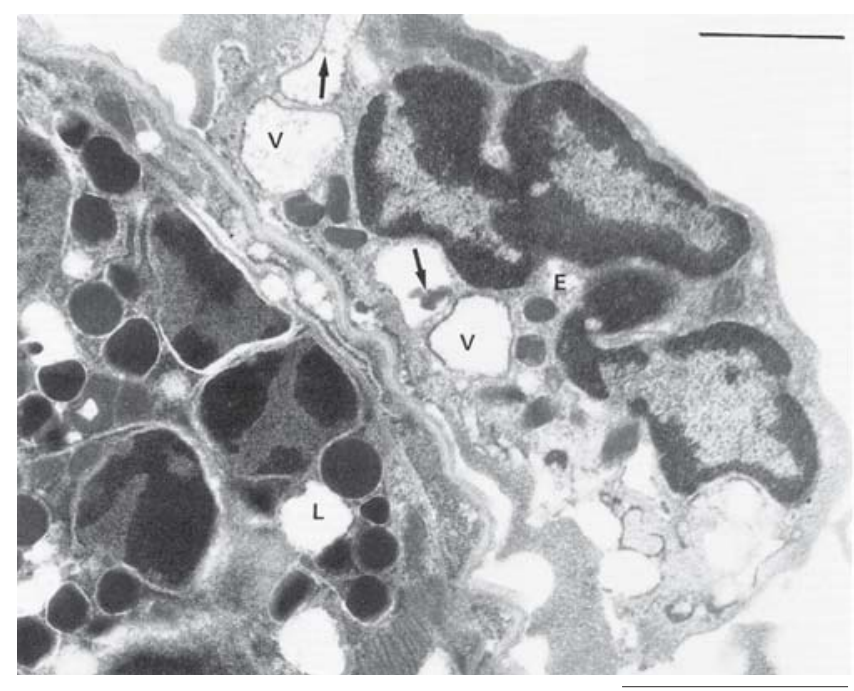

Figure 3. Electron micrograph of a degranulated eosinophil. Eosinophil (E) from a normal mouse, degranulated after adhesion to a Trichinella spiralis newborn larva (L) incubated with immune sera. ' $V$ ' indicates intracellular vacuoles with remnants of eosinophil granule content (arrows). Scale bar represents $1.25 \mu \mathrm{m}$. Reprinted, with permission, from Ref. [49].

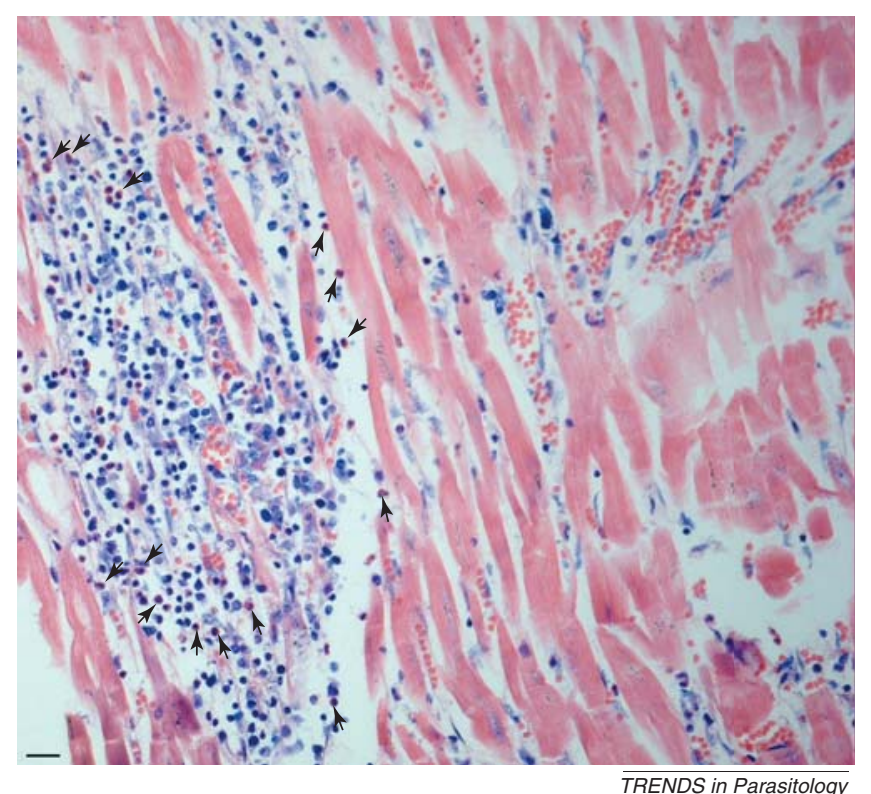

Figure 4. Eosinophils infiltrating the myocardial tissue of a trichinellosis patient. Eosinophils are indicated with arrows. This patient died of complications from trichinellosis. Tissue sample provided by Wanda Kociecka. Sample stained with May-Grunwald-Giemsa. Scale bar represents $50 \mu \mathrm{m}$. 
Table 1. Molecules involved in eosinophil differentiation, trafficking, survival and activation during Trichinella infection

\begin{tabular}{|c|c|c|c|}
\hline Molecule & Physiological role & Role in Trichinella infection & Refs \\
\hline IL-5 & $\begin{array}{l}\text { Differentiation and growth factor, eosinophil maturation and migration, } \\
\text { the inhibition of eosinophil apoptosis }\end{array}$ & $\begin{array}{l}\text { Protective }^{a} \\
\text { Protective in re-infection }\end{array}$ & $\begin{array}{l}{[8,45]} \\
{[46]}\end{array}$ \\
\hline IL-3 & Differentiation and growth factor, the inhibition of eosinophil apoptosis & - & {$[36]$} \\
\hline GM-CSF & Differentiation and growth factor, the inhibition of eosinophil apoptosis & - & [36] \\
\hline CD80 & Induction of eosinophilia & - & [12] \\
\hline CD86 & Induction of eosinophilia & - & [12] \\
\hline CCR3 & Eosinophil migration & Protective to newborn larvae & [14] \\
\hline$\beta 7$ integrin & Eosinophil migration & - & {$[19,20]$} \\
\hline Eotaxin-1 (CCL11) & Eosinophil trafficking & $-{ }^{\mathrm{b}}$ & [13] \\
\hline Eotaxin-2 (CCL24) & Eosinophil trafficking & - & [13] \\
\hline MBP & Eosinophil degranulation product & $\begin{array}{l}\text { Protective to newborn larvae, and } \\
\text { damaging to blood vessels and tissues }\end{array}$ & {$[59,60,64]$} \\
\hline ECP & Eosinophil degranulation product & $\begin{array}{l}\text { Protective to newborn larvae, and } \\
\text { damaging to blood vessels and tissues }\end{array}$ & {$[59,60,64]$} \\
\hline Ym1 & AAM product, Th2 induction & & [28] \\
\hline Arginase & AAM product, Th2 induction & & [29] \\
\hline
\end{tabular}

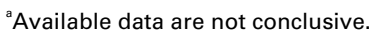

${ }^{\mathrm{b}}$ Disruption of the CCL11 gene does not alter the expulsion.

Abbreviations: $A A M$, alternatively activated macrophage; $C C L, C C$ chemokines ligand; $C C R$, $C C$ chemokine receptor; $C D$, cluster differentiation; $E C P$, eosinophil cationic protein; GM-CSF, granulocyte-macrophage colony-stimulating factor; IL, interleukin; MBP, major basic protein; Th, helper T cell; YM1, chitinase-like molecule. 